post-legislation in all countries. Proportions of samples containing $<0.1 \mathrm{ng} / \mathrm{ml}$ (i.e. undetectable) cotinine increased significantly $(\mathrm{RR}=1.63$; $95 \% \mathrm{CI}=1.45$ to 1.83 ), from $31.0 \%$ to $41.0 \%$. Although across the SES spectrum, there was no evidence of displacement of smoking into the home, socioeconomic inequality in the likelihood of samples containing detectable levels of cotinine increased $(\mathrm{RR}=1.10 ; 95 \% \mathrm{CI}=1.05$ to 1.16$)$. Among children from the poorest and most affluent families respectively, $96.9 \%$ and $38.2 \%$ of postlegislation samples contained detectable cotinine. Socioeconomic gradients at higher exposure levels remained unchanged. Among children from the poorest families, 1 in 3 samples contained greater cotinine concentrations than Scottish bar worker's samples prior to legislation $(3 \mathrm{ng} / \mathrm{ml})$. Smoking restrictions in homes and cars increased. However, little more than half (55.1\%) of children, and only $19.3 \%$ of children of smokers, lived in smoke-free homes following legislation. Significant socioeconomic patterning remained, with $26.3 \%$ and $72.0 \%$ of children from the poorest and most affluent families respectively living in a smoke-free home.

Conclusion Urgent action is needed to reduce inequalities in SHS exposure. Such action should include emphasis on reducing smoking in cars and homes.

\section{OP56 DIFFERENTIAL EFFECTS OF SMOKING CESSATION DURING PREGNANCY ON BIRTH WEIGHT IN A COHORT OF DISADVANTAGED WOMEN}

doi:10.1136/jech-2012-201753.056

${ }^{1,2} \mathrm{CB}$ Hayes, ${ }^{3} \mathrm{M}$ Kearney, ${ }^{3,4} \mathrm{C}$ Collins, ${ }^{3} \mathrm{H}$ O'Carroll, ${ }^{3} \mathrm{E}$ Wyse, ${ }^{3} \mathrm{M}$ Geary, ${ }^{5} \mathrm{CC}$ Kelleher 'Public Health and Primary Care, Trinity College Dublin, Dublin, Ireland; ${ }^{2}$ Public Health Department, Health Service Executive, Dublin, Ireland; ${ }^{3 R o t u n d a ~ H o s p i t a l, ~ D u b l i n, ~ I r e-~}$ land; ${ }^{4} / r i s h$ College of General Practitioners, Dublin, Ireland; ${ }^{5}$ School of Public Health, Physiotherapy and Population Science, University College Dublin, Dublin, Ireland

Background Smoking during pregnancy is recognized as the most important preventable risk factor. Maternal smoking accounts for $20-30 \%$ of low birth weight infants $(<2,500 \mathrm{~g})$, the most common adverse outcome in pregnancy. The objective of this study was to explore the effects of maternal smoking habits: stopping smoking in the first and second trimesters, continuing to smoke, number of cigarettes smoked and socio-demographic factors on infant birth weight.

Methods The study was a longitudinal cohort study of 1,000 pregnant smokers attending public hospital clinics in a disadvantaged catchment area at first pre-natal visit (V1), and assessed at 28-32 weeks (V2) and at one week after birth (V3) using an intervieweradministered questionnaire. The primary outcome variables were: change in smoking status based on self-reported response and urinary cotinine measurement for those who had quit. ANOVA was carried out to test for differences in mean birth weight. A multiple regression analysis with birth weight as the dependent variable was carried out on demographic and smoking characteristics and derived smoking category variables at V3: sustained quitters, continued smokers, successful quitters at V3 and intermittent quitters.

Results The mean difference in birth weight between continued smokers and sustained quitters was significant, (mean difference $=$ 233g, 95\% CI=60 - 406g, $\mathrm{p}=0.008$ ), as was the difference between continued smokers and intermittent quitters (mean difference $=$ 202g, 95\% CI $=17-386 \mathrm{~g}, \mathrm{p}=0.03$ ). Regression on baseline variables showed that only $2.4 \%$ of the variance $\left(\mathrm{R}^{2}\right)$ was explained by smoking characteristics; that is, number of smokers in the home other than self or partner $(p=0.008)$ and number of cigarettes smoked per day $(p=0.02)$. A second regression model showed gestation at delivery to be the best predictor of birth weight $\left(R^{2}=44.2\right)$. The number of cigarettes smoked at V2 explained an additional 2.1\% $(p<0.001)$ and being a sustained quitter $0.5 \%(\mathrm{p}=0.02)$.

Conclusion In this study a clear gradient was observed around smoking behaviour and birth weight with continued smokers having infants with lowest birth weights, sustained quitters the highest and intermittent quitters somewhere in between. The study also demonstrated that the negative effects of maternal smoking on birth weight are at least partly reversible. It thus showed a beneficial effect of quitting smoking for at least part of pregnancy and a link between passive smoking and birth weight. These findings are important for the delivery of targeted health promotion messages to smoking women in early pregnancy.

\section{Population Based Studies: Midlife}

\section{OP57 ALL-CAUSE AND CAUSE-SPECIFIC MORTALITY AMONG INDIVIDUALS WITH AND WITHOUT DIABETES IN ENGLAND AND SCOTLAND}

doi:10.1136/jech-2012-201753.057

VLZ Gordon-Dseagu, J Mindell, N Shelton. Department of Epidemiology and Public Health, UCL, London, UK

Background Although a growing body of evidence demonstrates an increase in cardiovascular disease (CVD) mortality among those with diabetes mellitus, the results related to other causes of death are less homogenous. The strength of the association between diabetes and mortality appears to differ by geographic location. The role that Body Mass Index (BMI) plays also requires further exploration. In the UK, one in 20 individuals is estimated to have diabetes. Therefore, even a small increase in mortality risk among those with diabetes, could result in a large number of deaths among those with the disease. This large general-population cohort study used data from England and Scotland to explore the associations between diabetes and risk of all-cause and cause-specific mortality, and examine the extent to which any increase was attributable to raised BMI. Methods Nationally-representative, cross-sectional data from 15 years of the Health Survey for England (HSE) (1994-2005) and Scottish Health Survey (SHeS) (1995, 1998 and 2003) were linked with mortality records up to the first quarter of 2011. Odds ratios (OR) and 95\% confidence intervals (CI) adjusted for age-group and sex (model 1), plus smoking status (model 2) and additionally for BMI category (model 3) were estimated using logistic and multinomial logistic regression. Participants mentioning cancer at baseline were excluded from the study.

Results Within this sample of 166,600 participants (5,131 with diabetes) there were 19,483 deaths (1,060 among those with diabetes, 18,423 without diabetes). All-cause mortality was greater among those with diabetes when adjusted for age, sex and smoking status (OR 1.52, 95\% CI 1.41-1.65), with no reduction when adjusting for BMI category (OR 1.49, 1.37-1.64). Cause-specific mortality among those with diabetes was raised for CVD (model 2 OR 1.73, 1.55-1.93), cancer $(1.24,1.08-1.43)$ and 'Other' (1.77, 1.54-2.04) with a non-significant increase for respiratory diseases (1.21, CI 0.99-1.47). Additional adjustment for BMI had a minimal impact upon the excess mortality found among those with diabetes: CVD (OR 1.69, 1.49-1.93), cancer $(1.24,1.05-1.45)$, 'Other' causes (1.75, 1.49-2.07), and respiratory diseases (1.16, 0.92-1.47). Survival was also lower among those with diabetes compared with those without the disease at baseline.

Conclusion Diabetes is associated with an excess of all-cause and cause-specific mortality from CVD, cancer, and 'Other' causes but probably not respiratory diseases. Increased BMI does not appear to be a mediating factor within the association between diabetes and cause-specific mortality.

OP58 IS THE EXCESS RISK OF MYOCARDIAL INFARCTION
AMONG PEOPLE WITH DIABETES FALLING OVER TIME?

doi:10.1136/jech-2012-201753.058 